Original Paper http://ajol.info/index.php/ijbcs http://indexmedicus.afro.who.int

\title{
Effet de la perte rapide volontaire de poids au cours d'un camp d'entraînement de deux semaines sur les paramètres hydro-électrolytiques et les performances des judokas de l'élite en Côte d'Ivoire
}

\author{
Jean-Paul KOUASSI ${ }^{1 *}$, N'guessan KOUAME ${ }^{2}$, Brigitte A. TONON ${ }^{1}$, \\ Polycarpe GOUTHON ${ }^{1}$, Basile K. NOUATIN ${ }^{1}$ et Antoine N. TAKO ${ }^{3}$ \\ ${ }^{1}$ Unité de Recherche Sport, Santé et Evaluation (UR/SSE), Institut National de la Jeunesse, de l'Education \\ Physique et du Sport (INJEPS), Université d'Abomey-Calavi, Porto-Novo, République du Bénin. \\ ${ }^{2}$ Laboratoire Sciences du Mouvement Humain et du Bien-Etre (LSMHBE), Institut National de la Jeunesse et \\ du Sport (INJS), Abidjan, République de Côte d'Ivoire. \\ ${ }^{3}$ Université Félix Houphouët-Boigny (UFHB), Cocody Abidjan, Côte d'Ivoire. \\ *Auteur correspondant ; E-mail : kouassijp1@yahoo.fr; Tel: 00225 55972799/00229 91288908
}

\section{RESUME}

Malgré les risques potentiels, des judokas pratiquent la perte rapide volontaire de poids en période de compétition. L'objectif de la présente étude était de mesurer les modifications induites par la perte rapide volontaire de poids (PRVP) sur les paramètres hydro-électrolytiques et les performances lors des tests de terrain, chez les judokas de Côte d'Ivoire pendant un camp d'entraînement (CE) de 15 jours. Pendant le CE, 33 judokas $(22,8 \pm 3,3$ ans) dont 25 hommes et 8 dames ont accepté de participer à cette étude prospective. Le poids, les concentrations des électrolytes urinaires, les performances à trois tests de terrain, la densité urinaire ont été évalués juste avant et à la fin du CE. A la fin du CE, les judokas pratiquants de la PRVP (groupe PP), ont perdu en moyenne $6,0 \pm 0,2 \%$ de leur poids $(\mathrm{p}<0,001)$ et étaient à un niveau de déshydratation sévère, alors que chez leurs homologues non pratiquants (groupe SP), le poids n'a pas varié ( $p>0,05$ ). Dans le groupe $\mathrm{PP}$, la concentration de $\mathrm{K}^{+}$a augmenté de 32,0\% $(\mathrm{p}=0,03)$ tandis que le ratio $\mathrm{Na} / \mathrm{K}$, les performances au «special judo fitness test », au saut en squat et le $\dot{V} \mathrm{O}_{2}$ max ont baissé respectivement de $31,4 \%(\mathrm{p}=0,04)$, $4,2 \%(\mathrm{p}<0,001), 6,5 \%(\mathrm{p}<0,0001)$ et $1,5 \%(\mathrm{p}<0,001)$. Dans le groupe SP, aucune modification significative n'a été observée $(\mathrm{p}>0,05)$. Au regard des risques que la PRVP présente pour la santé et la performance, tous les acteurs devraient comprendre la nécessité en cas de besoin, de procéder par une perte lente et progressive de poids en période de préparation aux compétitions.

(C) 2019 International Formulae Group. All rights reserved.

Mots clés: Judo, test de terrain, électrolytes, perte rapide volontaire de poids, déshydratation, Afrique subsaharienne.

\section{Effect of rapid weight loss during a two-week training camp on hydro electrolytic parameters and performance of elite judokas in Ivory Coast}

\section{ABSTRACT}

Despite the potential risks, judokas practice rapid weight loss during competitive periods. The objective of this study was to measure the changes induced by rapid weight loss (RWL) on hydro-electrolytic parameters and performance during field tests in judokas from Ivory Coast during a 15-day training camp (TC). During the 
TC, 33 judokas (22.8 \pm 3.3 years old) including 25 men and 8 women agreed to participate in this prospective study. Weight, urinary electrolyte concentrations, performance in three field tests, urinary density were assessed just before and at the end of the TC. At the end of the TC, the judokas practicing RWL (PP group), lost on average $6.0 \pm 0.2 \%$ of their weight $(\mathrm{p}<0.001)$ and were at a severe level of dehydration, while among their non-practicing counterparts (SP group), the weight did not change ( $\mathrm{p}>0.05$ ). In the PP group, the concentration of $\mathrm{K}+$ increased by $32.0 \%(\mathrm{p}=0.03)$ while the $\mathrm{Na} / \mathrm{K}$ ratio, performance in the special judo fitness test, squat jump and $\mathrm{VO}_{2}$ max decreased by $31.4 \%(\mathrm{p}=0.04), 4.2 \%(\mathrm{p}<0.001), 6.5 \%(\mathrm{p}<0.0001)$ and $1.5 \%(\mathrm{p}<0.001)$ respectively. In the SP group, no significant changes were observed $(\mathrm{p}>0.05)$. In view of the health and performance risks that the RWL poses, all stakeholders should understand the need, if necessary, to proceed with a slow and gradual weight loss during the preparation period for competitions.

(C) 2019 International Formulae Group. All rights reserved.

Keywords: Judo, field test, electrolytes, rapid weight loss, dehydration, sub-Saharan Africa.

\section{INTRODUCTION}

Dans les sports de combat comme le judo, pour éviter les blessures et donner une égalité de chance à tous, les compétitions se déroulent par catégorie de poids (Reale, 2018). Dans ce sport, à l'approche des compétitions, les judokas n'hésitent pas à perdre du poids, souvent en moins d'une semaine, pour combattre dans des catégories inférieures à celles qui correspondent à leur masse corporelle habituelle, par des procédés potentiellement dangereux pour leur santé (Artioli et al., 2016). Ils utilisent diverses méthodes à risque telles que le sauna, la privation volontaire de nourriture, d'eau et les combinaisons plastiques (Reale et al., 2017). $\mathrm{Au}$ cours de cette pratique qui s'intensifie deux à trois jours avant l'échéance, les athlètes perdent parfois plus de 5\% de leur poids (Artioli et al., 2016; Reale, 2018). La déshydratation que provoque cette pratique induit une dégradation des capacités physiologiques aérobies et anaérobies (Mc Dermott et al., 2017), ainsi qu'une perte des principaux électrolytes (sodium, potassium) nécessaires au bon fonctionnement des muscles (Casa et al., 2019).

En Afrique subsaharienne, des judokas pratiquent aussi la perte rapide volontaire de poids (RWL), alors que dans cet environnement chaud et humide, la situation s'aggrave très vite, du fait de la température ambiante et du taux d'humidité relativement élevés qui peuvent atteindre respectivement $35^{\circ} \mathrm{C}$ et $90 \%$ (Asseh et al., 2019; Kouakou et al., 2019). Le port du Judogi généralement cousu avec un tissu de coton épais, contribue aussi à accentuer les effets de la chaleur. Ces facteurs combinés provoquent la survenue précoce de l'état de déshydratation au cours de l'exercice physique. C'est dans ces conditions environnementales de contraintes thermiques élevées et de contexte de pratique difficile (Gouthon et al., 2015), que des judokas de l'élite de Côte d'Ivoire entreprennent la perte rapide volontaire de poids avant les compétitions nationales et internationales, en dépit des risques potentiels encourus.

A notre connaissance, les effets de la perte rapide volontaire de poids en période d'entraînement intensif sur les capacités physiologiques, musculaires, la performance au « Special Judo Fitness Test (SJFT) » et les indicateurs de l'état de bonne santé n'ont pas été évalués chez les judokas d'Afrique subsaharienne. Il est pourtant impérieux de réaliser de telles études, afin de faire prendre conscience aux judokas et aux encadreurs, des risques réels de cette pratique et d'optimiser l'entraînement préparatoire aux compétitions internationales dans un tel environnement.

L'objectif de cette étude était par conséquent de mesurer les effets de la perte rapide de poids volontaire pendant un camp d'entraînement de 15 jours, sur les paramètres hydro-électrolytiques, le $\dot{V} \mathrm{O}_{2}$ max et les performances à des tests de terrain chez les judokas de l'élite en Côte d' Ivoire.

\section{MATERIEL ET METHODES}

\section{Protocole de la recherche}

Cette étude prospective s'est déroulée pendant un camp d'entrainement (CE) de 15 jours, préparatoire au tournoi international de la ville d'Abidjan (TIVA), le plus grand 
organisé au judo en Côte D'Ivoire. Les mesures anthropométriques, les tests de terrain à savoir le «Special Judo Fitness Test (SJFT) », le Yoyo IRT2, le test de saut en squat (SS), puis le saut réactivité (SR) ainsi que la collecte d'urine ont été effectués avant et à la fin du CE. Tous les judokas ont été informés des objectifs de l'étude, puis ont donné librement leur consentement éclairé et écrit pour y participer. Ils ont obtenu la garantie que les résultats ne seraient utilisés en toute confidentialité. L'approbation de la Fédération Ivoirienne de Judo, ainsi que l'avis du Comité d'éthique de l'Institut National de la Jeunesse et des Sports $\left(\mathrm{N}^{\circ}\right.$ CMS/INJS 001/2017 du Comité d'éthique INJS, Centre de Médécine du Sport, 18 BP1717 Abidjan, felyan@yahoo.fr, Côte d'Ivoire) ont été requis et obtenus.

\section{Population d'étude}

L'effectif total était composé de 33 judokas seniors dont 25 hommes et huit dames, tous sélectionnés pour représenter la Côte d'Ivoire au tournoi international de la ville d'Abidjan (TIVA) de 2018. Parmi ces judokas, 13 étaient ceintures noires, 13 ceintures marron et sept ceintures bleues. Ces judokas participent régulièrement aux compétitions de niveau continental et deux sont de niveau olympique, huit autres ont été médaillés aux championnats d'Afrique et aux Jeux de la Francophonie. Pour cette étude, 18 pratiquants et 15 non-pratiquants de la PRVP ont été considérés comme appartenant respectivement aux groupes cas (PP) et contrôle (SP), après les deux premiers jours du camp d'entraînement.

\section{Mesures biochimiques, physiologiques et anthropométriques}

Les urines ont été collectées dans des tubes secs à usage unique et la température rectale $(\operatorname{Tr})$ mesurée avec des thermomètres automatiques MT 101R (Hangzou Sejoy, Chine), à usage personnel, en prenant les dispositions d'hygiène avant et après chaque opération. Les concentrations urinaires de sodium $\left(\mathrm{Na}^{+}\right)$, de potassium $\left(\mathrm{K}^{+}\right)$et des chlorures $\left(\mathrm{Cl}^{-}\right)$ont été déterminées avec un analyseur d'électrolytes 9180 (Roche, Suisse), puis le rapport $(\mathrm{Na} / \mathrm{K})$ a été calculé pour chaque participant. La concentration des électrolytes a été appréciée à partir des valeurs normales sur 24 heures (Frey et al., 2001) comprises pour le sodium entre 50 et 220 $\mathrm{mmol} / 24 \mathrm{~h}$, le potassium entre 25 et 130 $\mathrm{mmol} / 24 \mathrm{~h}$ et le chlorure entre 50 et 220 $\mathrm{mmol} / 24 \mathrm{~h}$. La densité urinaire (DU) a été mesurée avec un réfractomètre clinique PenUrine SG (Atago, Japon). Le statut hydrique a été déterminé à partir de la densité urinaire. Ainsi, un athlète était considéré comme normo-hydraté lorsque sa DU était $<1,020$. Il était déshydraté lorsque $1,020<\mathrm{DU}<1,030$. L'athlète était fortement déshydraté en cas de DU > 1,030 (Mc Dermott et al., 2017). La fréquence cardiaque $(\mathrm{Fc})$ et la pression artérielle (PA) des judokas ont été mesurées respectivement avec des cardiofréquencemètres Géonote (Decathlon, France) et un tensiomètre automatique 767 Plus (And UA, Japon). Le profil tensionnel des judokas a été déterminé à partir des valeurs de référence (Flint et al., 2019) telles que la pression artérielle systolique (PAS), seule composante de la pression artérielle concernée, a été considérée comme normale lorsqu'elle était comprise entre 120 et 129 $\mathrm{mmHg}$. Elle était normale haute quand elle était comprise entre 130 et $139 \mathrm{mmHg}$. L'athlète présentait une hypertension artérielle (HTA) de grade 1 (HTA1) lorsque la PAS était comprise entre 140 et $149 \mathrm{mmHg}$ et en HTA de grade 2 (HTA 2), lorsque sa valeur se situait entre 150 et $159 \mathrm{mmHg}$. L'indice physiologique de contrainte thermique (IPCT) a été calculé selon la formule: IPCT $=5\left(\mathrm{~T}_{\text {recf }}-\right.$ $\left.\mathrm{T}_{\text {reci }}\right)\left(39,5-\mathrm{T}_{\text {reci }}\right)^{-1}+5\left(\mathrm{Fc}_{\mathrm{f}}-\mathrm{Fc}_{\mathrm{i}}\right)\left(180-\mathrm{Fc}_{\mathrm{i}}\right)^{-}$ 1 , dans laquelle $\mathrm{T}_{\text {recf }}=$ température rectale finale (à la fin de l'exercice); $\mathrm{T}_{\text {reci }}=$ température rectale initiale (juste avant l'exercice); $\mathrm{Fc}_{\mathrm{f}}=$ fréquence cardiaque finale (à la fin de l'exercice); $\mathrm{Fc}_{\mathrm{i}}=$ fréquence cardiaque initiale. L'appréciation de l'IPCT a été faite selon l'échelle de trois niveaux (faible, modéré, élevé) proposée dans la littérature (Moran et al., 1998). La taille et le poids ont été mesurés respectivement en centimètre et en gramme, selon les techniques préconisées par Mac Dougall et al. (1988), avec une toise ZT-150A (Perlong Medical Equipement, Chine) et un pèse-personne automatique Xiangshian (Zhejiang, Chine). 
L'indice de masse corporelle (IMC) a été calculé selon la formule IMC $=\mathrm{P}(\mathrm{kg}) / \mathrm{T}^{2}(\mathrm{~m})$, dans laquelle $\mathrm{P}$ est le poids et $\mathrm{T}$, la taille. Le calcul du pourcentage de perte de poids (\%PP) a été effectué en utilisant la formule: \%PP = (perte de poids x 100) / poids avant le CE; dans laquelle la perte de poids = poids corporel avant le $\mathrm{CE}$ - poids corporel à la fin du CE. La température ambiante et l'humidité relative pendant les séances d'évaluation et au cours du CE ont été mesurées avec un appareil multifonction, c'est-à-dire hygromètre et thermomètre TP50 (ThermoPro, France). Chaque athlète disposait, selon sa demande, d'une ou deux bouteilles de 1,5 L d'eau courante à boire pendant chaque séance. La quantité d'eau bue à la fin de chaque séance d'entraînement a été déterminée par déduction, à partir de celle restante.

\section{Tests de terrain \\ Spécial Judo Fitness Test (SJFT)}

Le SJFT a été réalisé par groupe de trois judokas de la même catégorie de poids dont un Tori et deux Uke (Franchini et al., 2009). Tori effectuait une course navette en projetant chacun à son tour, les Uke pendant trois périodes respectives de $15 \mathrm{~s}, 30 \mathrm{~s}$ et $30 \mathrm{~s}$ par Ippon soe nage. Les Uke étaient à $6 \mathrm{~m}$ l'un de l'autre et Tori au milieu, à $3 \mathrm{~m}$ de chaque Uke. La fréquence cardiaque a été immédiatement lue dès la fin et une minute après le test. L'indice du SJFT (ISJFT) qui a permis de déterminer le niveau des capacités spécifiques des judokas en se référant à la table de classification de l'ISJFT, a été calculé à partir de l'équation suivante: ISJFT = (fréquence cardiaque dès la fin de l'exercice + fréquence cardiaque une minute après le test) / nombre total de projections (Franchini et al., 2009). L'indice de récupération cardiaque au SJFT $\left(\operatorname{IRC}_{\mathrm{SJFT}}\right)$ après une minute a été calculé en utilisant l'équation suivante: $\operatorname{IRC}_{\mathrm{SJFT}}=$ fréquence cardiaque (bpm) à la fin du SJFT fréquence cardiaque (bpm) 1 minute après le test (Franchini et al., 2009). Ainsi, plus la valeur de l'IRC $\mathrm{SJFT}_{\mathrm{S}}$ (bpm) est grande, meilleure est la récupération cardiaque de l'athlète.

\section{Yo-Yo intermittent recovery test 2 (Yo-Yo IRT2) et calcul du $\mathrm{V}^{\mathrm{O}_{2}} \max$}

Le $\dot{V} \mathrm{O}$ max a été indirectement estimé à partir du résultat au test Yo-Yo IRT2 (Bangsbo et al., 2008). Ce test s'effectue en course navette intermittente, sur une distance de $20 \mathrm{~m}$, avec une zone de récupération active de $5 \mathrm{~m}$. Au signal de l'enregistrement sonore qui sert de guide à la réalisation de l'épreuve, l'athlète parcourt la distance en aller et retour, puis marque une pause de $10 \mathrm{~s}$ en récupération active. A partir du nombre de paliers atteints par l'athlète, la distance parcourue a été déterminée et le $\dot{V} \mathrm{O}_{2} \max$ estimé avec l'équation suivante proposée par Bangsbo et al. (2008): $\dot{V} \mathrm{O}_{2} \max (\mathrm{mL} / \mathrm{min} / \mathrm{kg})=\mathrm{IRT} 2$ distance $(\mathrm{m}) \times 0,0136+45,3$.

\section{Tests de sauts}

Les performances au saut en squat (SS) et au saut réactivité (SR) ont été mesurées en utilisant le dispositif OptoJump Next (Microgate, Italie). Le SS a consisté à sauter le plus haut possible, à partir d'une position debout immobile, les genoux fléchis à $90^{\circ}$, mains sur les hanches. Le SR a été réalisé en 5 sauts, en recherchant la hauteur maximale et le temps de contact minimum au sol, à partir de la position debout, les réceptions étant souples et contrôlées. Le sujet effectuait une flexion des genoux à $90^{\circ}$ avant de sauter à nouveau.

\section{Camp d'entraînement}

Le camp d'entraînement (CE) organisé en 24 séances, était structuré en deux microcycles d'une semaine chacun. Deux séances quotidiennes ont été réalisées, le matin de $8 \mathrm{~h}$ à $10 \mathrm{~h}$ et l'après-midi de $17 \mathrm{~h}$ à 19 h. Le microcycle 1 était organisé en 6 séances sur une piste d'athlétisme le matin et 6 séances d'entraînement spécifique de judo l'après-midi. Le microcycle 2 était composé de 12 séances d'entraînement spécifique de judo.

Le microcycle 1 avait pour objectifs au plan énergétique, l'amélioration de la capacité de vitesse de course, de la vitesse maximale aérobie (VMA) et de l'endurance aérobie, qui ont occupé chacune quatre séances. Au plan musculaire, la force-vitesse et la force- 
endurance spécifique ont été visées respectivement pour $66,7 \%$ et $33,3 \%$ du temps de travail, soient huit et quatre séances. Au plan technico-tactique, le travail a porté sur les enchaînements techniques $(30 \%$ du temps), l'étude du Kumi-kata (20\% du temps), les liaisons debout-sol (20\% du temps), les renversements et les sorties d'immobilisation (10\% du temps), ainsi que sur la gestion des deux premières minutes de combat $(20 \%$ du temps). Au cours des séances sur piste, la vitesse au sprint et la VMA ont été recherchées en deux à trois séries de six sprints courts, sur $20,30,40$ puis 50 m courus à $100 \%$ de la vitesse maximale, avec une récupération complète, lorsque la source d'énergie ATP-CP était visée. Au total, trois séries de cinq sprints réalisées pendant quatre minutes, sur les mêmes distances, avec récupération incomplète, ont visé l'amélioration de la PMA.

Le microcycle 2 était consacré au plan énergétique à l'amélioration de la PMA et de la capacité ATP-CP pendant respectivement neuf (75\% du temps) et trois séances $(25 \%$ du temps). Au plan musculaire, la force-vitesse et la force-endurance spécifique ont occupé respectivement $41,6 \%$ et $58,3 \%$ du temps, soient cinq et sept séances chacune. L'aspect technico-tactique a porté, sur quatre séances (33,3\% du temps), sur la gestion des deux dernières minutes de combat, selon que l'athlète mène le combat ou qu'il est mené. Les enchaînements de trois, quatre ou cinq techniques de jambes, hanche ou épaule qui finissent avec le Tokui waza (mouvement favori) avec la liaison debout-sol, ont aussi fait l'objet de quatre séances. Au sol, le travail a porté sur les clés, les étranglements, les immobilisations et a occupé 4 séances.

Les séances d'entraînement spécifique de judo au cours du cycle avaient une durée moyenne de deux heures. Chaque séance comportait les six parties suivantes: 1) le salut et la prise en main en cinq minutes; 2) l'échauffement en $25 \mathrm{~min}$; 3) l'apprentissage technico-tactique en $30 \mathrm{~min}$; 4) les Randoris ou combats libres pendant $45 \mathrm{~min}$; 5) la relaxation et le retour au calme en $15 \mathrm{~min}$; 6) le salut et la reprise en main pendant cinq minutes. Toutes les séances d'entraînement spécifique de judo (microcycles 1 et 2) comportaient des séquences de 15 min de renforcement musculaire (pompes, squats, abdominaux) essentiellement basées sur l'amélioration de la force-endurance.

\section{Analyse statistique}

Les résultats ont été traités avec le logiciel Statistica (StatSoft. Inc., version 12.0). La normalité des distributions a été vérifiée avec le test de Kolmogorov Smirnov et l'homogénéité des variances a été testée avec le test de Levène. L'ANOVA à deux facteurs (Groupe x Temps de mesure) a été utilisée pour rechercher les interactions entre les variables groupe et temps de mesure. Parce que les distributions des variables étaient toutes non-normales, le test de rang de Wilcoxon a été utilisé pour tester l'effet temps de mesure dans chaque groupe. Les comparaisons intergroupes ont été réalisées avec le test U de Man Whitney. Le niveau de signification des tests statistiques a été fixé à $\mathrm{p}$ $<0,05$.

\section{RESULTATS}

L'échantillon de cette étude était constitué de 18 judokas de niveau d'instruction universitaire, 12 de niveau secondaire et trois de niveau primaire. Dans les deux groupes, les poids légers et moyens au nombre de 31, constituaient $94 \%$ de l'échantillon. Les ceintures noires et marron au nombre de 26 représentaient 78,8\% de l'effectif. Au cours du CE, tous les 18 judokas du groupe PP, qui représentaient $54,5 \%$ de l'effectif, ont perdu $6,0 \pm 0,2 \%(p<0,0001)$ de leur poids corporel.

Au début du CE, sept judokas dans chacun des groupes PP et SP, représentant respectivement $38,8 \%$ et $46,6 \%$ des athlètes étaient déshydratés (Tableau 1). A la fin du CE, tous les 18 judokas (100\%) du groupe PP l'ont été, tandis qu'il n'y a eu que cinq judokas déshydratés $(33,3 \%)$ dans le groupe SP. Au début du CE, il n'y avait pas de différence significative $(p>0,05)$ entre les deux groupes par rapport à la concentration des électrolytes. En revanche, à la fin du CE, la concentration $\mathrm{du} \mathrm{K}^{+}$urinaire a significativement augmenté de 32\% ( $\mathrm{p}=$ 0,03 ), alors que le rapport $\mathrm{Na} / \mathrm{K}$ a baissé de $31,4 \%(\mathrm{p}=0,04)$ dans le groupe PP 
uniquement. Toutes les modifications des électrolytes observées dans le groupe SP n'étaient pas significatives ( $p>0,05)$.

$\mathrm{Au}$ début du $\mathrm{CE}$, les valeurs de repos de fréquence cardiaque et de pression artérielle systolique étaient normales et similaires dans les deux groupes. A la fin du $\mathrm{CE}$, dans le groupe $\mathrm{PP}$, une élévation de 11 mmHg représentant $8,4 \%(\mathrm{p}<0,001)$ de la PAS de repos a été constatée (Tableau 2), avec deux et trois judokas qui présentaient respectivement une HTA de grade 1 et de grade 2. Dans le groupe SP, les modifications des paramètres cardio-vasculaires enregistrées à la fin du CE n'étaient pas significatives ( $p$ > $0,05)$.

Au début du CE, il n'y avait pas de différence significative $(\mathrm{p}>0,05)$, entre les deux groupes, en ce qui concerne le $\dot{V} \mathrm{O}_{2} \max$, les performances aux tests de sauts et au SJFT. En revanche, à la fin du CE dans le groupe $\mathrm{PP}$, le $\dot{\mathrm{V}} \mathrm{O}_{2}$ max, la hauteur du SS et le nombre total de projections ont significativement baissé respectivement de $1,5 \%(\mathrm{p}<0,001)$, de 6,5\% ( $<<0,0001)$ et de $3,4 \%(\mathrm{p}<0,001)$ (Tableau 2). Dans ce même groupe, une tendance à la baisse de $13 \%$ de

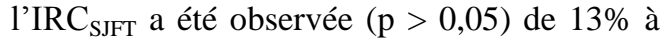
la fin du $\mathrm{CE}$, et une augmentation significative de 4,2\% (p < 0,001) de l'ISJFT a été constatée (Tableau 2). Par contre dans le groupe SP (Tableau 2), à la fin du CE, un accroissement significatif de la hauteur du SS de 4,3\% (p < 0,001) a été constaté (Tableau 2). Dans le groupe SP, les performances au SJFT et les valeurs des autres paramètres n'ont pas été significativement affectées à la fin du CE ( $p$ > $0,05)$.

Pendant le CE (Tableau 3) les judokas des groupes PP et SP ont travaillé respectivement à 78,4 $\pm 2,0 \%$ et $78,9 \pm 2,0 \%$ de leur Fc max théorique. La température moyenne et l'hygrométrie étaient en moyenne de $29^{\circ} \mathrm{C}$ et $78 \%$ d'humidité pendant le $\mathrm{CE}$ et les séances de prise de mesures. L'IPCT qui était modéré dans le groupe $\mathrm{SP}(6,6 \pm 0,4)$ était en moyenne significativement plus élevé dans le groupe PP $(7,3 \pm 0,4 ; \mathrm{p}<0,001)$. Pendant le CE, la quantité d'eau bue était significativement plus faible $(\mathrm{p}<0,001)$ dans le groupe PP que dans le groupe SP.

Tableau 1: Concentration des électrolytes urinaires et statut hydrique au cours du camp. d'entraînement chez des judokas de l'élite en Côte d'Ivoire $(n=33)$.

\begin{tabular}{cccc}
\hline \multicolumn{2}{c}{ Groupe PP $(\mathrm{n}=18)$} & \multicolumn{2}{c}{ Groupe SP $(\mathrm{n}=15)$} \\
M1 & M2 & M1 & M2 \\
\hline $165,8 \pm 90,4$ & $144,3 \pm 76,4$ & $133,9 \pm 46,1$ & $164,2 \pm 77,9$ \\
$57,0 \pm 27,7$ & $75,4 \pm 30,6^{*}$ & $60,6 \pm 32,6$ & $63,4 \pm 39,7$ \\
$156,9 \pm 88,7$ & $146,6 \pm 82,6$ & $131,8 \pm 67,1$ & $173,0 \pm 71,5$ \\
$3,5 \pm 1,8$ & $2,4 \pm 1,8^{*}$ & $2,9 \pm 2,2$ & $3,5 \pm 3,0$ \\
$1,019 \pm 0,008$ & $1,032 \pm 0,008^{* * *}$ & $1,016 \pm 0,009$ & $1,018 \pm 0,016^{\dagger \dagger \dagger}$ \\
$11(62,1)$ & $0(0,0)$ & $8(53,3)$ & $10(66,7)^{*}$ \\
$7(38,9)$ & $18(100,0)$ & $7(46,7)$ & $5(33,3)^{*}$ \\
\hline
\end{tabular}

Les nombres dans les cases représentent les valeurs moyennes \pm écarts types; Les nombres suivis de parenthèses représentent les fréquences absolues et les pourcentages; PRVP: perte rapide volontaire de poids; Na: concentration urinaire de sodium; $\mathrm{K}$ : concentration urinaire de potassium; $\mathrm{Cl}$ : concentration urinaire de chlorure; $\mathrm{Na} / \mathrm{K}$ : rapport sodium/potassium. $\mathrm{PP}$ : groupe des judokas pratiquants de la PRVP; SP: groupe des judokas non-pratiquants de la PRVP; M1: mesures effectuées avant le camp d'entraînement; M2: mesures effectuées à la fin du camp d'entraînement; *: différence avec M1, significative à p < 0,$05 ; * * *$ : différence avec $\mathrm{M} 1$, significative à $\mathrm{p}<0,001 ;{ }^{\dagger \dagger}$ : différence avec $\mathrm{PP}$, significative à $\mathrm{p}<0,001$. 
Tableau 2: Modifications des paramètres anthropométriques, physiologiques, des performances aux tests de terrain au cours d'un camp d'entraînement chez des judokas de l'élite en Côte d'Ivoire ( $\mathrm{n}=$ $33)$.

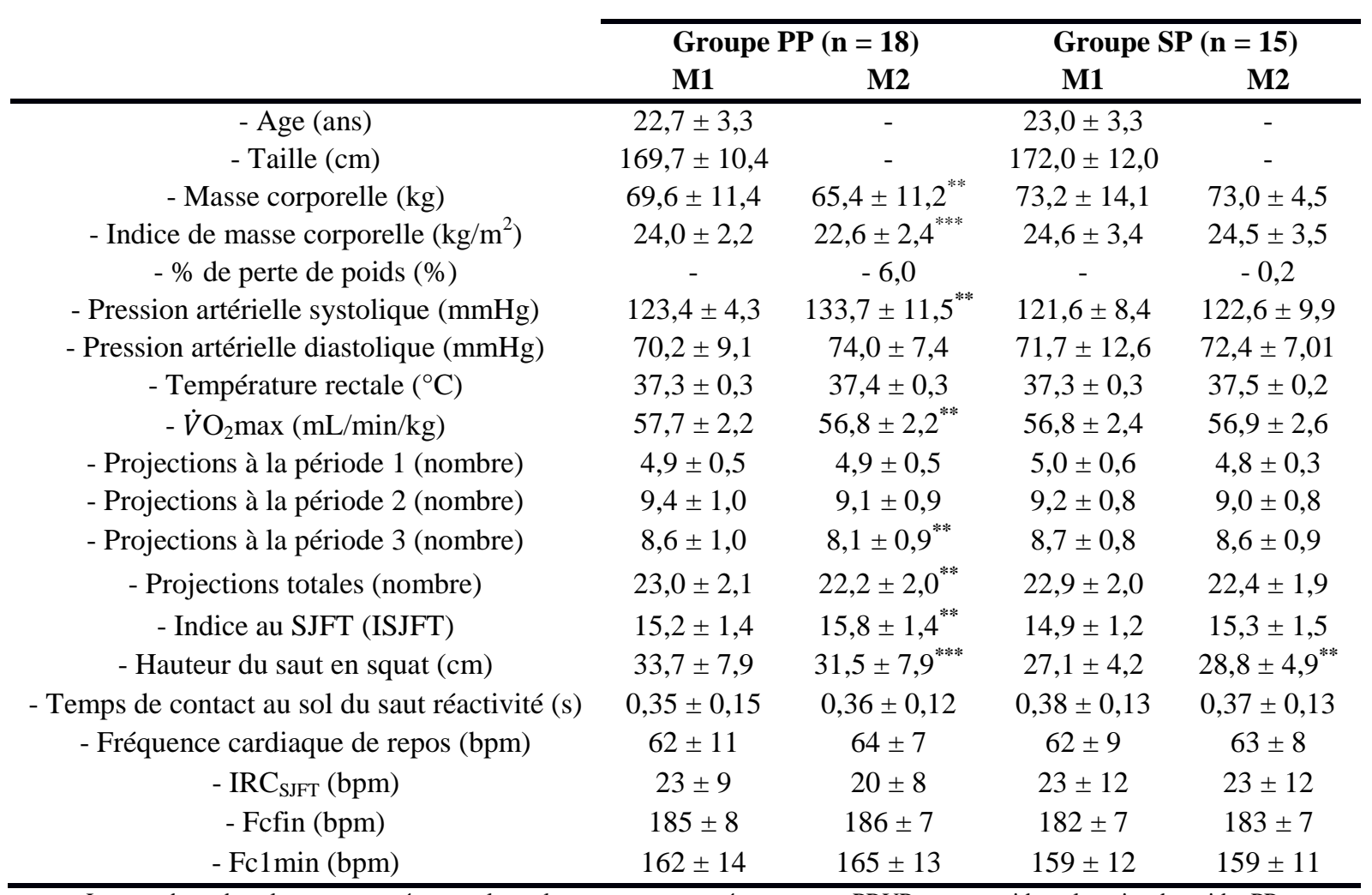

Les nombres dans les cases représentent les valeurs moyennes \pm écarts types; PRVP: perte rapide volontaire de poids; PP: groupe des Judokas pratiquants de la PRVP; SP: groupe des judokas non-pratiquants de la PRVP; SJFT: Special Judo Fitness Test; M1: mesures effectuées avant le camp d'entraînement; M2: mesures effectuées à la fin du camp d'entraînement; Fcfin: fréquence cardiaque mesurée juste à la fin du SJFT; Fc1mn: fréquence cardiaque mesurée une minute après la fin du SJFT; $\dot{V} \mathrm{O}_{2}$ max: consommation maximale d'oxygène, estimée indirectement par les résultats au test Yoyo IRT2; IRC $\mathrm{SIFT}_{\text {: indice de }}$ récupération cardiaque calculé à partir de la différence entre Fcfin et Fc1min; **: différence avec M1, significative à p < 0,01 .

Tableau 3: Comparaisons des données collectées dans les groupes de pratiquants et de nonpratiquants de la PRVP au cours du camp d'entraînement $(\mathrm{n}=33)$.

\begin{tabular}{ccccccc}
\cline { 2 - 6 } & \multicolumn{3}{c}{ Groupe PP $(\mathrm{n}=18)$} & \multicolumn{3}{c}{ Groupe SP $(\mathrm{n}=15)$} \\
\cline { 2 - 7 } & $\begin{array}{c}\text { Moyenne } \\
\text { microcycle 1 }\end{array}$ & $\begin{array}{c}\text { Moyenne } \\
\text { microcycle 2 }\end{array}$ & $\begin{array}{c}\text { Moyenne } \\
\text { du cycle }\end{array}$ & $\begin{array}{c}\text { Moyenne } \\
\text { microcycle 1 }\end{array}$ & $\begin{array}{c}\text { Moyenne } \\
\text { microcycle 2 }\end{array}$ & $\begin{array}{c}\text { Moyenne } \\
\text { du cycle }\end{array}$ \\
\hline - Fcr (bpm) & $62 \pm 10$ & $63 \pm 7$ & $62 \pm 8$ & $61 \pm 8$ & $62 \pm 8$ & $62 \pm 8$ \\
- Fcmoy (bpm) & $155 \pm 3$ & $154 \pm 4$ & $155 \pm 2$ & $156 \pm 3$ & $154 \pm 4$ & $155 \pm 3$ \\
- Fcmax (bpm) & $197 \pm 4$ & $197 \pm 4$ & $197 \pm 4$ & $196 \pm 3$ & $196 \pm 3$ & $196 \pm 3$ \\
- \% Fcmax (\%) & $79,3 \pm 2,0$ & $78,4 \pm 2,0$ & $78,8 \pm 1,7$ & $79,3 \pm 1,9$ & $78,4 \pm 2,5$ & $78,9 \pm 2,0$ \\
- IPCT & $7,0 \pm 0,5$ & $7,6 \pm 0,5^{* * *}$ & $7,3 \pm 0,4$ & $6,5 \pm 0,5^{\dagger \dagger}$ & $6,6 \pm 0,5^{\dagger \dagger \dagger}$ & $6,6 \pm 0,4^{\dagger \dagger \dagger}$ \\
- Eau bue (L) & $1,5 \pm 0,3$ & $1,0 \pm 0,3^{* * *}$ & $1,2 \pm 0,3$ & $1,7 \pm 0,2$ & $1,7 \pm 0,1^{\dagger \dagger \dagger}$ & $1,7 \pm 0,1^{\dagger \dagger \dagger}$ \\
\hline
\end{tabular}

Les nombres dans les cases représentent les moyennes \pm écarts types; PRVP: perte rapide volontaire de poids; PP: groupe des judokas pratiquants de la PRVP; SP: groupe des judokas non pratiquants de la PRVP; IPCT: indice physiologique de contrainte thermique; Fréquence cardiaque de repos; Fcmax: Fréquence cardiaque maximale théorique; Fcmoy: fréquence cardiaque moyenne d'effort; \%Fc: pourcentage de la fréquence cardiaque maximale; ${ }^{\dagger \dagger}$ : différence avec PP, significative à $\mathrm{p}<$ 0,$01 ;{ }^{* * *}:$ différence avec la moyenne du microcycle 1 , significative à $\mathrm{p}<0,001 ; ;^{\dagger \dagger}$ : différence avec PP, significative à $\mathrm{p}<$ 0,001 . 


\section{DISCUSSION}

Cette étude s'appuie sur l'hypothèse selon laquelle, pendant un camp d'entraînement de deux semaines, la perte rapide volontaire de poids infléchit les performances à des tests de terrain, influence négativement les paramètres hydroélectrolytiques et physiologiques chez les judokas de l'élite de Côte d'Ivoire. Les résultats ont montré que la performance au «special judo fitness test», le $\dot{V} \mathrm{O}_{2} \max$, la hauteur de saut en squat et le rapport $\mathrm{Na} / \mathrm{K}$ ont baissé, alors que la concentration de $\mathrm{K}^{+}$a augmenté, du fait de la perte rapide volontaire de poids. La durée de deux semaines du camp d'entraînement a permis d'observer quotidiennement les judokas in-situ et de constater la constante dans leur conduite. Les résultats peuvent donc être considérés comme fiables, puisque les dispositions ont été prises pour respecter les exigences méthodologiques. Les résultats sont par ailleurs valables pour l'ensemble des judokas de l'élite de Côte d'Ivoire, étant donné que l'échantillon a été constitué avec les meilleurs judokas qui représentent ce pays aux différentes compétitions internationales. Le fait de n'avoir pas pris en compte les variations de l'eau corporelle et du pourcentage de graisse, pourrait limiter la portée des résultats qui méritent d'être confirmés dans d'autres pays d'Afrique subsaharienne où le judo est bien pratiqué.

Pendant le camp d'entraînement, les 18 judokas $(54,5 \%)$ pratiquant la perte rapide de poids ont perdu en moyenne $6 \%$ de leur poids. Dans ce groupe, huit athlètes ont en outre perdu près de $10 \%$ de leur poids. Le pourcentage de poids perdu est supérieur à la perte maximale de 4,5\% récemment observée au Taekwondo sur une période trois à quatre semaines (Seyhan, 2018). Dans les sports de percussion, comme le Taekwondo, l'amplitude de la perte rapide volontaire de poids semble généralement plus faible que celle des sports de préhension. En effet dans les disciplines de percussion, la perte rapide volontaire de poids n'offre qu'un léger avantage en termes de facilité dans la mobilité et les déplacements (Reale et al., 2017). Par contre, dans les sports de préhension comme le judo où il faut soulever, projeter, contrôler le poids de l'adversaire, être plus lourd, donne un avantage physique notable (Reale, 2018). Toutefois, une étude a rapporté au Taekwondo des pratiques semblables à ce qui s'observe dans les sports de préhension comme le judo (Fortes et al., 2017a). Dans cette discipline, la perte de poids qui représente en moyenne 5\% du poids (Artioli et al., 2016), peut parfois atteindre plus de 10\% (Fortes et al., 2017b). La perte moyenne de $6 \%$ observée en deux semaines dans la présente étude, n'est donc pas exceptionnelle. Une perte lente et progressive de poids est pourtant la meilleure option quand un athlète veut changer de catégorie de poids (Artioli et al., 2016). Mieux, pour préserver la santé de l'athlète, la perte de poids doit être encadrée et suivie par des professionnels de la santé, surtout qu'il est recommandé de ne pas perdre plus de $1,5 \%$ de son poids par semaine (Reale et al., 2017). Les judokas de la présente étude ont pourtant perdu plus de $6 \%$ de leur le poids, sans assistance médicale, en s'exposant aux risques potentiels (Artioli et al., 2016).

L'une des conséquences de cette pratique, à la fin du camp d'entraînement, a été l'augmentation de $8,4 \%$ de la pression artérielle systolique dans le groupe des pratiquants de la PRVP, avec deux et trois judokas qui présentaient respectivement une hypertension de grade 1 et de grade 2 (Flint et al., 2019). En effet, la déshydratation peut provoquer une baisse du volume plasmatique, puis une augmentation de la viscosité sanguine, qui induit une élévation de la résistance à l'écoulement du sang et conduit à une augmentation de la pression artérielle (Guezennec, 2011). L'augmentation de cette pression, semble traduire un des premiers signes de perturbation cardiovasculaire que peut occasionner une perte rapide volontaire de poids (Mountjoy et al., 2018). 
A la fin du camp d'entraînement, tous les 18 judokas qui ont pratiqué la PRVP, étaient fortement déshydratés. Il est admis que quand les athlètes des sports de combat se déshydratent pour «faire le poids», ils perdent aussi des électrolytes (Casa et al., 2019). Dans la présente étude, l'augmentation de la concentration du $\mathrm{K}^{+}$urinaire et la baisse du $\mathrm{Na} / \mathrm{K}$ témoignent de la perte de l'eau intra et extracellulaire, puis d'une fuite du potassium. En effet, pendant la déshydratation, la baisse $\mathrm{du}$ volume de l'eau extracellulaire provoque une diffusion du liquide intracellulaire vers le milieu extracellulaire; le potassium, électrolyte du milieu intracellulaire passe alors dans le liquide extracellulaire (Kenefick, 2018) et est excrété dans les urines. Il est donc impérieux, d'optimiser la réhydratation de tous les athlètes pendant les camps d'entraînement intensif, par des solutions enrichies en électrolytes (Casa et al., 2019).

A la fin du camp d'entraînement, la hauteur du saut en squat a baissé de 6,5\% dans le groupe des pratiquants de la perte de poids alors que dans celui des non-pratiquants, elle a augmenté de 4,3\%. La détérioration de la capacité de saut dans la présente étude pourrait être attribuée à deux facteurs, la déshydratation et la fatigue musculaire (Bishop et Spencer, 2004). En effet, la majorité des pratiquants de la perte de poids ont consacré leur temps de repos aux exercices supplémentaires. Le manque de repos a ainsi compromis la récupération favorable à la surcompensation des réserves énergétiques musculaires (Sedeaud et al., 2018). De ce fait, ces judokas n'ont pas tiré grand avantage du programme d'entraînement sur ce plan, par rapport à leurs homologues du groupe des non-pratiquants, qui au contraire, ont enregistré une amélioration significative de la performance à ce test de saut. Des baisses similaires de hauteurs de sauts ont été observées chez des judokas africains, chez qui, la restriction alimentaire du Ramadan a eu les mêmes conséquences musculaires (Aloui et al., 2012).
Le $\dot{V} \mathrm{O}_{2}$ max a aussi baissé à la fin du camp d'entraînement chez les pratiquants de la perte rapide volontaire de poids, alors que chez les non-pratiquants il n'a pas varié. En effet, la déshydratation des pratiquants de la perte rapide volontaire de poids doit avoir provoqué d'une part, la réduction du volume plasmatique et d'autre part une redistribution $\mathrm{du}$ volume sanguin vers la peau, pour l'élimination de la chaleur (Casa et al., 2019). Cette situation a certainement provoqué une baisse du volume sanguin veineux, infléchissant le remplissage ventriculaire et le volume d'éjection systolique. La baisse du volume d'éjection systolique a induit une réduction du débit cardiaque (Guezennec, 2011) facteur déterminant du $\dot{V} \mathrm{O}_{2} \max$ (Bassett et Howley, 2000). Il faut rappeler que la déshydratation peut induire l'affaiblissement des systèmes tampons et une augmentation de l'acidose musculaire, provoquant une baisse de la capacité des muscles à synthétiser les phosphagènes (Bishop et Spencer, 2004). Dans ces conditions, la capacité de récupération diminue et l'aptitude à soutenir un effort intermittent, surtout un effort de nature aérobie baisse (McDermott et al., 2017).

L'indice au special judo fitness test élevé au départ, a augmenté de $4 \%$ chez les pratiquants de la PRVP à la fin du camp d'entraînement, indiquant que la performance au SJFT a baissé. Cette baisse s'est traduite par la réduction du nombre total de projections dans ce groupe et la baisse de $13 \%$ de l'indice de récupération cardiaque $\left(\mathrm{IRC}_{\mathrm{SJFT}}\right)$. D'autres auteurs ont constaté une baisse de la performance au SJFT chez des judokas africains, après une baisse progressive de $1,7 \%$ du poids en une semaine (Lalia et al., 2019) et une baisse plus rapide de 4,17\% après une semaine (Abedemaleck et al., 2015). Dans ces deux études, les deux modèles de perte de poids ont entraîné une baisse de la performance au SJFT, une réduction de la capacité de récupération cardiaque et du nombre de projections comme dans la 
présente étude. Cependant d'autres auteurs sont parvenus à des résultats différents, après avoir observé des pertes rapides de poids de $10 \%$ chez des judokas européens, en deux semaines. En effet, ces auteurs ont rapporté que la perte rapide volontaire de poids n'avait pas provoqué de changement au niveau de l'indice au SJFT chez les pratiquants de la PRVP, mais chez les non pratiquants une amélioration significative de ce paramètre en termes de baisse de l'indice SJFT a été constatée (Fortes et al., 2017b). Les conclusions de toutes ces études sont identiques aux observations de la présente étude, puisqu'elles révèlent toutes que la perte rapide volontaire de poids compromet la performance à des tests de terrain et donc potentiellement la performance sportive. Il n'est donc pas évident que cette pratique apporte une amélioration de la performance du judoka (Fortes et al., 2017b).

Les résultats de la présente étude peuvent servir de base pour la sensibilisation des judokas, afin qu'ils évitent de s'engager dans cette pratique puisque le risque d'infléchissement de la performance sportive et de dégradation de leur état de santé est potentiellement accru au terme de la PRVP réalisée en ambiance chaude et humide. Ces résultats qui ont mis en évidence les effets potentiellement nocifs de la perte rapide volontaire de poids sur la performance à des tests de terrain, les paramètres physiologiques et hydro-électrolytiques, permettent également aux entraîneurs d'améliorer le suivi des athlètes en période de préparation, pour une plus grande efficacité des programmes d'entraînement.

\section{Conclusion}

Cette étude a été entreprise sur la base de l'hypothèse selon laquelle la perte rapide volontaire de poids influence négativement les paramètres hydro-électrolytiques, anthropométriques et les performances à des tests de terrain chez les judokas de l'élite de Côte d'Ivoire, pendant un camp d'entraînement. A la fin du camp, les athlètes qui ont pratiqué la perte rapide volontaire de poids ont tous été sévèrement déshydratés, avec une baisse des performances aux trois tests de terrain retenus, et une élévation de la pression artérielle systolique. Cette pratique présente donc un double risque pour les judokas, à savoir pour leur santé et leur capacité de performance. Au regard des résultats obtenus, il est nécessaire d'envisager un meilleur encadrement des judokas de Côte d'Ivoire par des professionnels de la santé, afin que les recommandations officielles en la matière soient respectées. Tous les acteurs du judo doivent en effet comprendre et accepter de tendre vers une perte lente et progressive de poids en période de préparation des compétitions. Les prochains travaux devront explorer dans cet environnement chaud, l'effet de la PRVP sur les paramètres immunitaires et inflammatoires des judokas de l'élite. Il faudra aussi intégrer aux protocoles de recherche, la réalisation de tests d'évaluation de la fatigue musculaire en plus à celle normalement induite par l'entraînement intensif.

\section{CONFLIT D'INTERETS}

Les auteurs déclarent ne pas avoir de conflit d'intérêt.

\section{CONTRIBUTIONS DES AUTEURS}

J-PK et BNN : Elaboration du projet de recherche. N'GK, PG : Validation du projet de recherche. J-PK, NK, BAT, CD et ANT: Collectes de données. J-PK, BAT et BNN : Analyse statistique. J-PK, NK, BAT, PG, BNN, CD et ANT: Interprétations des données. J-PK : Rédaction du manuscrit. PG, $\mathrm{CD}$ et ANT: relecture et correction du manuscrit.

\section{REMERCIEMENTS}

Les auteurs remercient le Directeur Général de l'Institut National des Sports d'Abidjan (INJS) pour toute l'assistance dans l'analyse des échantillons et les commodités offertes lors de la réalisation de cette étude. 


\section{REFERENCES}

Reale R. 2018. Acute weight management in combat sports: pre-weight-in weight loss, post weight-in recovery and competition nutrition strategies. Sports. Sci. Exch., 29(183): 1-6.

Artioli GG, Saunders B, Iglesias RT, Franchini E. 2016. It's time to ban rapid weight loss from combat sports. Sports. Med., 46(11): 1579-1584. DOI: 10.1007/s40279-016-0541-x

Reale R, Slater G, Burke LM. 2017. Individualized dietary strategies for Olympic combat sports: Acute weight loss, recovery and competition nutrition. Eur. J. Sports. Sci., 17: 727-740. DOI: 10.1080/17461391.2017.1297489

McDermott BP, Anderson SA, Armstrong LE, Casa DJ, Cheuvront SN, Cooper L. 2017. National Athletic Ttrainers' Association Position Statement: Fluid replacement for the physically active. $J$. Athl. Train., 52(9): 877-895. DOI: 10.4085/1062-6050-52.9.02

Casa D, Cheuvront SN, Galloway SD, Shirreffs SM. 2019. Fluid needs for training, competition, and recovery in track and field athletes. Int. J. Sport. Nutr. Exerc. Metab., 29(2): 175-180. DOI: 10.1123/ijsnem.2018-0374

Asseh EE, Ake-Assi E, Koffi KJ. 2019. Diversité biologique et influence des changements climatiques sur la distribution géographique de quelques espèces d'Acanthaceae en Côte d'Ivoire. Int. J. Biol. Chem. Sci., 13(2): 676-692. DOI: 10.4314/ijbs.v13i2.9

Kouakou KJ, Gogbeu SJ, Sika AE, Yao KB, Bounakhla M, Zahry F. 2019. Caractérisation physico-chimique des horizons de surface de sols à maraîchers dans la ville d'Abidjan (Côte d'Ivoire). Int. J. Biol. Chem. Sci., 13(2):1193-1200. DOI: $10.4314 /$ ijbs.v13i2.47

Gouthon P, Kouassi JP, N'Guessan K, BioNigan I, Tonon B, Nouatin B. 2015. Knowledge, attitude and practice regarding muscular reinforcement among judokas in developing countries: Case study of Republic of Benin. Ann. Appl. Sport. Sci., 2(3): 11-22. DOI: 10.18869/acadpub.aassjournal.3.2.11

MacDougall JD, Wenger HA, Green HJ. 1988. Evaluation Physiologique de L'Athlète de Haut Niveau. Edition Vigot: Paris.

Flint AC, Conell C, Ren X, Banki NM, Chan SL, Rao VA. 2019. Effect of systolic and diastolic blood pressure on cardiovacular outcomes. N. Eng. J. Med., 381(3): 243251. DOI: $10.1056 /$ NEJMoa1803180

Moran DS, Shitzer A, Pandolf KB. 1998. A physiological strain index to evaluate heat stress. Am. J. Physiol., 44: 129-134.

Frey J, Daudon M, Raby N, Augereau C, Dechaux M, Diehl JL. 2001. Valeurs sémiologiques des paramètres biochimiques urinaires. Annal. Biol. Clin., 59(1): 13-25.

Franchini E, Del Vecchio FB, Sterkowicz S. 2009. A special judo fitness test classificatory table. Arch. Budo., 5(1): 127-129.

Bangsbo JF, Iaia M, Krustrup P. 2008. The yo-yo intermittent recovery test. A useful tool for evaluation of physical performance in intermittent sports. Sports. Med., 38(1): 37-51.

Seyhan S. 2018. Evaluation of rapid weight loss practices of taekwondo athletes and their effects. J. Educ. Train. Study, 6(10): 213-218. DOI: 10.11114/jets.v6i10.3663

De Sousa Fortes L, de Vasconcelos GC, de Vasconcelos Costa BD, Paes PP, Franchini E. 2017a. Effect of 10\% weight loss on simulated taekwondo match performance: a randomized trial. J. Exerc. Rehabil., 13(6):659-665. DOI: 10.12965/jer.1735134.567

Fortes LS, Costa BDV, Paes PP, Cyrino ES, Vianna JM, Franchini E. 2017b. Effect of rapid weight loss on physical performance in judo athletes: is rapid 
weight loss a help for judokas with weight problems? Int. J. Perform. Anal. Sport., 17(5): 763-773. DOI: $10.1080 / 24748668.2017 .1399323$

Guezennec CY. 2011. Les boissons de l'effort: Bases physiologiques de leurs utilisations et composition. Cahier. Nutr. Diét., 46: S46-S53. DOI: 10.1016/S0007-9960(11)70009-8

Mountjoy M, Sundgot-Borgen, Burke LM, Ackerman KE, Blauwet C, Constantini N. 2018. IOC consensus statement on relative energy deficiency in sport (REDS): 2018 update. Br. J. Sports. Med., 52: 687-697. DOI: 10.1123/IJSNEM.20180136

Kenefick RW. 2018. Drinking strategies: planned drinking versus drinking to thirst. Sports. Med., 48(1): S31-S37. DOI: $10.1007 / \mathrm{s} 40279-017-0844-6$

Aloui A, Chtourou H, Masmoudi L, Chaouachi A, Chamari K, Souissi N. 2013. Effect of ramadan fasting on male judoka's performances in specific and non-specific judo tasks. Biol. Rythm. Res., 44(4): 645-654. DOI: 10.1080/09291016.2012.722454

Sedeaud A, Sène JM, Krantz N, Saulière G, Moussa I, Toussaint JF. 2018.
L'importance de la quantification de la charge d'entrainement: exemple d'un modèle. Sci. Sport., 33(1): 22-32. DOI: 10.1016/j.scispo.2017.06.011

Bassett DR, Howley ET. 2000. Limiting factors for maximum oxygen uptake and determinant of endurance performance. Med. Sci. Sports. Exerc., 32(1): 70-84. DOI: $\quad 10.1097 / 00005768-200001000-$ 00012

Bishop D, Spencer M. 2004. Determinants of repeated-sprint ability in well-trained team sport athletes and endurancetrained athletes. J. Sports. Med. Phys. Fitness., 44: 1-7.

Lalia C, Alrawi AR, Abdel B, Asli H, Othman B. 2019. Effects of caloric restriction on anthropmetrical and specific performance in highly-trained university judo athletes. Physical Education of Students, 23(1): 30-36. DOI: 10.15561/20755279.2019.0105

Abedelmalek S, Chtourou H, Souissi N, Tabkar Z. 2015. Caloric restriction effect on pro-inflammatory cytokines, growth hormone, and steroid hormone concentrations during exercise in judokas. Oxid. Med. Cell. Longev., 1: 18. DOI: $10.1155 / 2015 / 809492$. 